\title{
Immunotherapy and non-small cell lung cancer
}

\author{
Letizia Laera ${ }^{1}$, Silvia Novello
}

\begin{abstract}
Lung cancer is the leading cause of cancer mortality, and non-small cell lung cancer (NSCLC) accounts for more than $85 \%$ of all lung cancers. Immunotherapy has brought a paradigm shift to the treatment of NSCLC. Immune checkpoint inhibitors have emerged as one of the main new therapeutic options for patients with advanced NSCLC. This brief review focuses on analyzing the biological rationale and early clinical data available concerning immunotherapeutic strategies, and more specifically, programmed cell death-1 (PD-1) inhibitors and programmed cell death-ligand 1 (PD-L1) inhibitors.
\end{abstract}

Key words: lung cancer, immunotherapy, programmed cell death-1 (PD-1) inhibitors, programmed cell deathligand 1 (PD-L1) inhibitors

\section{Introduction}

Lung cancer remains one of the most common cancers worldwide and approximately $70 \%$ of these patients present with advanced disease. Almost $80 \%$ are classified as non-small cell lung cancer (NSCLC), and 70\% of these have non-squamous histology subtype [1]. Current treatment strategies for advanced NSCLC include platinumbased doublet chemotherapy based on histology and targeted agents based on specific altered genomic mutations (epidermal growth factor receptor [EGFR] mutations found in $10-20 \%$ of NSCLC Caucasian tumors, anaplastic lymphoma kinase [ALK] found in $4-5 \%$ of NSCLC or ROS1 translocation/re-arrangements found in $1 \%$ of NSCLC) [2]. Platinum-based chemotherapy offers a higher response rate and prolonged survival in $15-30 \%$ of cases [3]. In the absence of genomic mutations, secondline treatment more frequently consists of taxane-based chemotherapy with a low response rate [4]. However, the majority of these patients do not attain a durable disease control, and the 5-year survival rate remains low.

"UO Oncologia "Sandro Pitigliani", Nuovo Ospedale di Prato "Santo Stefano", Azienda USL Toscana Centro, Prato, Italy. ${ }^{2}$ AOU S. Luigi Gonzaga, Università degli Studi di Torino, Torino, Italy. Correspondence to:

Letizia Laera,

UO Oncologia "Sandro Pitigliani", Nuovo Ospedale

di Prato "Santo Stefano", Azienda USL Toscana Centro,

Via Suor Niccolina Infermiera 20, 59100 Prato, Italy.

Phone: +390574 805304

E-mail: letizia.laera@uslcentro.toscana.it

CANCER BREAKING NEWS 2017;5(3):6-10

DOI: $10.19156 / \mathrm{cbn} .2017 .0052$
The ability of lung cancer to evade immune-surveillance is a result of the production by tumor cell (TC) of immunosuppressive chemokines, loss of major histocompatibility complex (MHC) antigen expression, and increased number of T-regulatory (Treg) cells in the tumor microenvironment [5]. Recently, connections between checkpoint pathways and cancer immunity have presented new opportunities for clinical development, by using the immune system against cancer cells through $\mathrm{T}$ cell activation and regulation in the lymphatic tissue (through cytotoxic T-lymphocyte-associated protein 4 (CTLA-4) inhibition) or in the peripheral tissue (programmed death (PD-1), programmed death ligand (PD-L1) checkpoint inhibition) [5]. Novel approaches involving checkpoint inhibitors can reactivate immune responses, and these have been shown to improve survival benefits in various cancer patients, including advanced squamous and nonsquamous NSCLC.

\section{Immune checkpoint inhibition and immune checkpoint inhibitors}

An important mechanism of immune resistance involves immune-inhibitory pathways called immune checkpoints, which normally intervene in the maintenance of immune tolerance and reduce collateral tissue damage [5]. CTLA-4 is expressed in T cells and primarily regulates CD4+ $\mathrm{T}$ cells and enhances the immunosuppressive activity of Treg cells, which are needed in the early stages of T-cell activation, counteracting the activity of the Tcell co-stimulatory receptor $\mathrm{CD} 28$ by competing for its B7 ligand. In contrast, PD-1 is a negative regulator of 
T-cell activity that limits the activity of $\mathrm{T}$ cells at a variety of stages of the immune response when it interacts with its two ligands PD-L1 and PD-L2. When engaged with the ligands through phosphatase activity, PD-1 inhibits kinase signaling pathways that normally lead to T-cell activation. Unlike CTLA-4, which is primarily believed to regulate immune responses early in T-cell activation, PD-1 is primarily believed to inhibit effector T-cell activity in the effector phase within tissue and tumors. CTLA-4 and PD-1/PD-L1 are the two immune checkpoint receptors most widely studied in the context of clinical cancer immunotherapy. Immunologic checkpoint blockade with antibodies that target the CTLA-4 and the PD-1/PD-L1 have shown efficacy in various malignancies [6]. Anti-PD-1 agents and anti-PD-L1 agents have demonstrated high efficacy for the treatment of advanced NSCLC.

\section{PD-1 checkpoint inhibitors}

Nivolumab, a fully human IgG4 monoclonal antagonist antibody to PD-1, was the first anti-PD1 agent to receive US Food and Drug Administration (FDA) approval. In two phase III trials nivolumab demonstrated improved overall survival (OS) compared to docetaxel in patients with squamous and non-squamous cell histologies after first-line platinum-doublet chemotherapy $[7,8]$. The first trial, CheckMate 017 trial (NCT01642004), enrolled 272 patients with previously treated advanced squamous NSCLC randomized to receive nivolumab $(3 \mathrm{mg} / \mathrm{kg}$ intravenously every 2 weeks) or docetaxel $\left(75 \mathrm{mg} / \mathrm{m}^{2}\right.$ intravenously every 3 weeks) [7]. The primary endpoint of OS was achieved with a median value of 9.2 versus 6 months in the nivolumab arm versus control arm. The one-year survival rate was $42 \%$ with nivolumab versus $24 \%$ with docetaxel. Secondary endpoints, objective response rate (ORR) and duration of response (DoR), were also higher in the nivolumab arm. Positive results were then shown for non-squamous histology in the CheckMate 057 trial (NCT01673867). Nivolumab was recently evaluated against standard platinum-doublet chemotherapy in chemotherapy-naïve stage IV NSCLC patients in an open-label, randomized phase III trial called CheckMate 026 (NCT02041533) [9]. The primary endpoint was progression-free survival (PFS) in patients with a PD-L1 expression of at least 5\%. However, it was not achieved. OS and ORRS was similar in the two arms: OS was 14.4 months for nivolumab versus 13.2 months for chemotherapy with a 1 -year OS rate of $56 \%$ and $54 \%$, respectively; ORRs appeared $26 \%$ compared to $34 \%$ with platinum-doublet chemotherapy. The high crossover rate $(64.2 \%)$ in the chemotherapy arm compared to $43.6 \%$ in the nivolumab arm could partially explain the lack of OS benefit, together with a suboptimal patient selection and inclusion in terms of PD-L1 expression. Clinical trials are currently evaluating the combination of nivolumab with chemotherapy, biologic drugs, and other immunotherapeutic agents.

One interesting strategy is the association of PD- 1 checkpointblockade with aCTLA-4 inhibitor(ipilimumab) [10]. Based on the encouraging results of a phase I study that analyzed this combination, a phase III trial called CheckMate 227 (NCT02477826) has been designed. This four-arm study, which is currently recruiting, compares platinum-doublet chemotherapy plus nivolumab versus nivolumab/ipilimumab in PD-L1-expressing advanced NSCLC and platinum-doublet/nivolumab combination versus nivolumab/ipilimumab combination in PD-L1negative ones [11, 12].

Pembrolizumab is another humanized antagonist antibody to PD-1. In NSCLC, pembrolizumab is the first-line treatment if the cancer overexpresses PD-L1 $(\geq 50 \%)$, in the absence of mutations in EGFR or in ALK, then it can be used as second-line treatment for advanced tumors expressing PD-L1 on at least $1 \%$ of TCs [13]. Several KEYNOTE trials have demonstrated efficacy and safety. The phase I dose expansion KEYNOTE-001 trial (NCT01295827) evaluated the safety and antitumor activity of pembrolizumab in advanced lung cancer [14]. A subsequent phase II/III study (KEYNOTE-010) compared two dosing levels of pembrolizumab $(2 \mathrm{mg} / \mathrm{kg}$ and $10 \mathrm{mg} / \mathrm{kg}$ ) with docetaxel in 1034 patients with previously treated NSCLC and assessed the role of PD-L1 expression. The results met their primary endpoint of higher OS and PFS, both in pembrolizumab $2 \mathrm{mg} / \mathrm{kg}$ and 10 $\mathrm{mg} / \mathrm{kg}$ every 3 weeks arms. Also, pembrolizumab groups showed better outcomes in patients with high PD-L1 expression (tumor proportion score of $50 \%$ or greater).

These data were the first to prospectively show the utility of PD-L1 as a biomarker [15]. The recently completed phase III KEYNOTE-024 trial compared pembrolizumab monotherapy (200 mg intravenously every 3 weeks) with investigator's choice platinum-doublet chemotherapy in untreated metastatic NSCLC in the absence of genomic mutations with PD-L1 expression on at least $50 \%$ of tumor cells. Final data confirmed that PFS, OS, ORRs and $\mathrm{mDoR}$ wasa prolonged for patients treated in the pembrolizumab arm [16]. Other clinical studies are ongoing to evaluate the combination of pembrolizumab with chemotherapy, biologic drugs, and other immune-modulating agents. The KEYNOTE-021 phase II trial compared platinum/pemetrexed-doublet therapy with platinum/pemetrexed/pembrolizumab triplet therapy in 123 patients 
with PD-L1-unselected, advanced, chemotherapy-naïve non-squamous NSCLC. Very interesting data showed significantly better ORR with the triplet therapy compared to the doublet (55\% and 29\%, respectively). PFS was also significantly higher in the study arm [17]. The phase III trial, KEYNOTE-189 (NCT02578680) is ongoing.

\section{PD-L1 checkpoint inhibitors}

Atezolizumab is the first humanized, engineered IgG1 monoclonal antibody against the first protein programmed death ligand 1 (PD-L1) to receive FDA approval for metastatic treated and untreated advanced NSCLC. The two most important open-label phase II multicenter trials, POPLAR (NCT01903993) [18] and BIRCH (NCT02031458) [19] showed higher benefit in term of OS, PFS, and ORR in the atezolizumab arm when compared to single-agent docetaxel. The open-label randomized phase III OAK trial (NCT02008227) confirmed positive results and decreed the role of atezolizumab in previously treated NSCLC [20]. This study enrolled patients with advanced squamous or non-squamous nonsmall cell lung cancer who had progressed on one to two prior chemotherapy regimens to receive atezolizumab or docetaxel. OS in the PD-L1-enriched population resulted higher in the atezolizumab arm being 15.7 months compared with docetaxel (10.3 months). Patients with low or undetectable PD-L1 expression also had survival benefit with atezolizumab with a median OS (mOS) of 12.6 months versus 8.9 months with chemotherapy and results were similar in both squamous and non-squamous histologies, regardless of PD-L1 expression. As seen in the POPLAR and BIRCH studies, data showed a favorable safety profile in the immunotherapy arm. There are many ongoing clinical trials in pretreated, metastatic NSCLC with atezolizumab as monotherapy or in combination with other agents to evaluate the efficacy and safety of atezolizumab in neoadjuvant and adjuvant settings (NCT02927301, NCT02716038). Studies are also investigating the combination of atezolizumab with external beam radiation in patients with locally advanced lung cancer (NCT02444741) [21].

Durvalumab is a high-affinity IgG1 human monoclonal antibody that blocks the interaction of PD-L1 with the PD-1 and B7-1molecules. It does not change the interaction between PD-1 and PD-L2, it releases the inhibition of immune response, without inducing antibody-dependent cell-mediated cytotoxicity (ADCC) and complement-dependent cytotoxicity (CDC). First phase I/II durvalumab as a prior or subsequent treatment in patients with stage IIIB/IV was presented the first time at the European Society for Medical Oncology (ESMO) 2016
(NCT02572843) and showed benefit in term of OS and ORR for patients receiving durvalumab as second-line treatment both in subgroup with high PD-L1 expression and in those with low expression; similar higher 1-year OS rates were observed in patients receiving third-line or higher therapy in the high and low expression groups. The phase II, single-arm, ATLANTIC Study (NCT02087423) investigated durvalumab in third-line or higher NSCLC and showed similar data [22]. The primary endpoint was ORR and resulted higher in cohort 2 (patients with PD-L1 > 25\%) and cohort 3 (patients with PD-L1 >90\%). The results from the randomized, double-blind, phase 3 PACIFIC trial assessed the antitumor activity of durvalumab in NSCLC stage III patients after chemoradiation therapy. The primary endpoint PFS was 16.8 months in the durvalumab arm versus 5.6 months with placebo with a good safety profile [23]. Several clinical trials are currently underway, exploring the combination of durvalumab with other agents such as tremelimumab, mocetinostat (a histone deacetylase inhibitor), chemotherapy and others $[24,25]$. Avelumab is a fully humanized anti-PD-L1 IgG1 which retains a native Fc region enabling ADCC via engagement of the innate immune system. The first phase I trial (NCT01772004) evaluated the safety and the antitumor activity in advanced solid tumors and results from pretreated NSCLC cohort demonstrated encourage data of ORR and PFS. Another phase $\mathrm{Ib}$ trial investigated the activity of avelumab in untreated patients with NSCLC not selected for PD-L1 expression. The results showed that among those 75 patients with $>3$ months of follow-up, there was an ORR of $19 \%$ including 1 complete response and 12 responses ongoing. In additional $45 \%$ of cases, stable disease was observed, reaching a disease control rate of $64 \%$. A randomized phase III study is evaluating the role of avelumab compared with platinum-based doublet chemotherapy in untreated patients with advanced disease (JAVELIN Lung 100; NCT02576574) and a phase III multicenter (JAVELIN Lung 200; NCT02395172) clinical trial is randomizing patients to avelumab versus docetaxel in patients with advanced NSCLC previously treated with platinum-based doublet chemotherapy.

\section{Patient selection: are there biomarkers for immune checkpoint inhibition?}

Patient selection for checkpoint inhibitors and identification of predictive biomarkers for immune therapies are currently active areas of research. PD-L1 expression by immunohistochemistry (IHC) has been suggested to predict response to PD-1 and PD-L1 inhibitors but some factors, such as the lack of standardized assay and cut-off 
points, timing of assessing (i.e., archival or fresh material), and disparity in different clinical trial results make this biomarker the "most famous, but less appreciated". The overexpression of PD-L1 by IHC has demonstrated to improve clinical outcomes in patients treated with anti-PD-1 and anti-PD-L1-directed therapy. Clearly, PD-L1 staining is an imperfect marker, whose negativity does not exclude a response to immunotherapy, as well as the roughly half of patients who are "non-responders" despite high tumor PD-L1 levels. Smoking history has also been associated with an increased overall response rate to PD-1, and for this reason, smoking status is sometimes considered as a surrogate marker for mutational density. Predictive biomarkers that can direct the rational use of PD-1/PD-L1 checkpoint inhibitors are crucial not only for a better selection of those patients who really benefit from their use but also because a biomarker-driven selection of immunotherapy responders and non-responders would reduce the financial burden for health systems associated with these innovative treatments. Refining existing biomarkers and identifying novel ones will be key to ensuring the effective and safe use of these agents [26]. Somatic mutations in the cancer genome are a consequence of multiple processes including deficiencies in DNA repair, endogenous and exogenous carcinogen exposure, and enzymatic alterations in DNA [27]. Most of these mutations derive from passenger genes and lead to translation of novel peptide epitopes, or neoantigens, which are presented by the MHC on the surface of malignant cells. Theoretically, the presence of neoantigens enhances the immunogenicity of the tumor by eliciting $\mathrm{T}$ cell repertoires that recognize these antigens as "foreign" and infiltrate the tumor microenvironment. Recent advances in sequencing technology allow for whole exome interrogation and qualitative calculation of the total number of mutations per coding region or total mutation burden (TMB). Several recent studies have evaluated TMB as a candidate biomarker for immunotherapeutic approaches in both melanoma and lung cancer. TMB seems promising as its predictive utility has been demonstrated across multiple tumor types although further validation will be needed in larger studies [28].

\section{Conclusions and future directions}

There is still a way to go in determining the role of immunotherapy in NSCLC, with issues to overcome and new targets to develop. Different settings (other than advanced disease) need more data: in locally advanced tumors, studies suggested that immunotherapy may be an effective adjuvant therapy in patients with stage III disease after standard treatment, but they need further investigation. Significant efforts are being made into evaluating novel approaches combining PD-1 or PD-L1 inhibitors with other treatment, such as chemotherapy, targeted therapies, radiation and other immunotherapeutic agents. The optimal duration of immunotherapy in patients with durable responses remains unclear. Currently, data from only one prospective trial have been presented, which showed that for patients still on nivolumab at one year, PFS was significantly improved for those treated continuously versus those stopping (CheckMate 153). The feasibility of retreatment for patients who were previously responders but who went off therapy for various reasons also needs to be further explored, as well as radiological interpretation with new immune-related criteria, different from the traditional Response Evaluation Criteria In Solid Tumors (RECIST) v1.1 criteria.

\section{Acknowledgments}

The Authors thank Ray Hill, an independent medical writer, who provided native English editing and journal styling on behalf of HPS.

\section{Conflicts of Interest}

The Authors declare there are no conflicts of interest in relation to this article. 


\section{References}

1. Travis WD, Brambilla E, Riely GJ. New pathologic classification of lung cancer: relevance for clinical practice and clinical trials. J Clin Oncol 2013;31(8):992-1001.

2. Aisner DL, Marshall CB. Molecular pathology of nonsmall cell lung cancer: a practical guide. Am J Clin Pathol 2012;138(3):332-46.

3. Schiller JH, Harrington D, Belani CP et al. Comparison of four chemotherapy regimens for advanced non-small-cell lung cancer. N Engl J Med 2002;346(2):92-8.

4. Weiss JM, Stinchcombe TE. Second-line therapy for advanced NSCLC. Oncologist 2013;18(8):947-53.

5. Pardoll DM. The blockade of immune checkpoints in cancer immunotherapy. Nat Rev Cancer 2012;12(4):252-64.

6. Postow MA, Callahan MK, Wolchok JD. Immune Checkpoint blockade in cancer therapy. J Clin Oncol 2015; 33(17):1974-82.

7. Brahmer J, Reckamp KL, Baas $P$ et al. Nivolumab versus docetaxel in advanced squamous-cell non-small-cell lung cancer. N Engl J Med 2015;373(2):123-35.

8. Borghaei H, Paz-Ares L, Horn L et al. Nivolumab versus docetaxel in advanced nonsquamous non-small-cell lung cancer. N Engl J Med 2015;373(17):1627-39.

9. Socinski M, Creelan B, Horn L et al. NSCLC, metastatic CheckMate 026: A phase 3 trial of nivolumab vs investigator's choice (IC) of platinum-based doublet chemotherapy (PT-DC) as first-line therapy for stage IV/recurrent programmed death ligand 1 (PD-L1)-positive NSCLC [Abstract]. Ann Oncol 2016;27(suppl_6):LBA7_PR.

10. Tanvetyanon T, Gray JE, Antonia SJ. PD-1 checkpoint blockade alone or combined PD-1 and CTLA-4 blockade as immunotherapy for lung cancer? Expert Opin Biol Ther 2017;17(3):305-12.

11. Hellmann MD, Rizvi NA, Goldman JW et al. Nivolumab plus ipilimumab as first-line treatment for advanced non-small-cell lung cancer (CheckMate 012): results of an open-label, phase 1, multicohort study. Lancet Oncol 2017;18(1):31-41.

12. Hellmann M, Ramalingam S, Reck M et al. An open label randomized Phase III trial of nivolumab or nivolumab plus ipilimumab vs platinum doublet chemotherapy (PT-DC) in patients with chemotherapy-naïve stage IV or recurrent non-small cell lung cancer (NSCLC) (CheckMate 227) [Abstract]. J Immunother Cancer 2015;3(2):P154.

13. Sul J, Blumenthal GM, Jiang X et al. FDA Approval Summary: Pembrolizumab for the treatment of patients with metastatic non-small cell lung cancer whose tumors express programmed death-ligand 1. Oncologist 2016; 21(5):643-50.

14. Garon EB, Rizvi NA, Hui R et al. Pembrolizumab for the treatment of non-small-cell lung cancer. N Engl J Med 2015;372(21):2018-28.

15. Herbst RS, Baas P, Kim DW et al. Pembrolizumab versus docetaxel for previously treated, PD-L1-positive, advanced non-small-cell lung cancer (KEYNOTE-010): a randomised controlled trial. Lancet 2016;387(10027):1540-50.
16. Reck M, Rodriguez-Abreu D, Robinson AG et al. Pembrolizumab versus chemotherapy for PD-L1-positive non-small-cell lung cancer. N Engl J Med 2016;375(19): 1823-33.

17. Langer CJ, Gadgeel SM, Borghaei $\mathrm{H}$ et al. Carboplatin and pemetrexed with or without pembrolizumab for advanced, non-squamous non-small-cell lung cancer: a randomised, phase 2 cohort of the open-label KEYNOTE-021 study. Lancet Oncol 2016;17(11):1497-508.

18. Fehrenbacher L, Spira A, Ballinger $M$ et al. Atezolizumab versus docetaxel for patients with previously treated non-small-cell lung cancer (POPLAR): a multicentre, open-label, phase 2 randomised controlled trial. Lancet 2016;387(10030):1837-46.

19. Wakelee H, Patel JD, Heist R et al. ORAL01.04: Phase II trial of atezolizumab for patients with PD-L1-selected advanced NSCLC (BIRCH): updated efficacy and exploratory biomarker results: Topic: medical oncology. J Thorac Oncol 2016;11(11S):S251-S2.

20. Rittmeyer A, Barlesi F, Waterkamp D et al. Atezolizum$\mathrm{ab}$ versus docetaxel in patients with previously treated non-small-cell lung cancer (OAK): a phase 3, openlabel, multicentre randomised controlled trial. Lancet 2017;389(10066):255-65.

21. Seetharamu N, Preeshagul IR, Sullivan KM. New PD-L1 inhibitors in non-small cell lung cancer-impact of atezolizumab. Lung Cancer: Targets Ther 2017;8:67-78.

22. Garassino M, Vansteenkiste J, Kim J-H et al., editors. Durvalumab in $\geq 3$ rd-line locally advanced or metastatic, EGFR/ALK wild-type NSCLC: Results from the phase 2 ATLANTIC study [Abstract]. 2016 World Conference on Lung Cancer. Abstract PL04a; 2016.

23. Antonia SJ, Villegas A, Daniel D et al. Durvalumab after chemoradiotherapy in stage III non-small-cell lung cancer. N Engl J Med 2017;377(20):1919-29.

24. Juergens R, Hao D, Laurie S et al. MA09. 03 Cisplatin/pemetrexed+ durvalumab+/-tremelimumab in pts with advanced non-squamous NSCLC: A CCTG Phase IB Study-IND. 226 [Abstract]. J Thorac Oncol 2017;12(1): S392-S3.

25. Mok T, Schmid P, De Castro G et al. P2. 06-022 First-line durvalumab plus tremelimumab vs platinum-based chemotherapy for advanced/metastatic NSCLC: Phase 3 NEPTUNE study [Abstract]. J Thorac Oncol 2017;12(1):S1084.

26. Gridelli C, Ardizzoni A, Barberis M et al. Predictive biomarkers of immunotherapy for non-small cell lung cancer: results from an Experts Panel Meeting of the Italian Association of Thoracic Oncology. Transl Lung Cancer Res 2017;6(3):373-86.

27. Alexandrov LB, Nik-Zainal S, Wedge DC et al. Signatures of mutational processes in human cancer. Nature 2013;500(7463):415-21.

28. Voong KR, Feliciano J, Becker D et al. Beyond PD-L1 testing-emerging biomarkers for immunotherapy in non-small cell lung cancer. Ann Transl Med 2017;5(18):376. 